\title{
Polysomnographic Sleep and Attentional Deficits in Traumatized North Korean Refugees
}

\author{
Jooyoung Lee' \\ Sehyun Jeon (iD) ${ }^{2}$ \\ Somin Kim (iD) \\ Yumin Seo' \\ Jinme Park ${ }^{3}$ \\ Yu Jin Lee ${ }^{4}$ \\ Seog Ju Kim' \\ 'Department of Psychiatry, \\ Sungkyunkwan University College of \\ Medicine, Samsung Medical Center, \\ Seoul, Republic of Korea; ${ }^{2}$ Department of \\ Psychiatry, Korea University Anam \\ Hospital, Seoul, Republic of Korea; \\ ${ }^{3}$ Department of Psychology, University of \\ Konstanz, Konstanz, Germany; \\ ${ }^{4}$ Department of Psychiatry and Center \\ for Sleep and Chronobiology, Seoul \\ National University, College of Medicine \\ and Hospital, Seoul, Republic of Korea
}

Correspondence: Seog Ju Kim

Department of Psychiatry, Sungkyunkwan University School of Medicine, Samsung

Medical Center, 8I Ilwon-ro, Kangnam-gu, \#0635I, Seoul, I35-710, Korea

Tel +82-2-3410-3583

Fax +82-2-3410-0050

Email ksj7I26@skku.edu
Introduction: Attentional deficits and sleep problems are common in refugees who have experienced trauma. In the present study, we used polysomnography (PSG) to investigate the relationship between attentional deficits and objective measures of sleep structure in traumatized North Korean refugees.

Methods: We recruited 32 North Korean refugees (mean age $=33.78 \pm 14.33$ years) and 39 South Korean participants (mean age $=35.03 \pm 11.08$ years). Sustained attention and divided attention were assessed using the Computerized Attention Test. We conducted an overnight PSG to objectively assess sleep structure. The participants also completed the Beck Depression Inventory (BDI), Beck Anxiety Inventory (BAI), Impact of Event ScaleRevised (IES-R), Epworth Sleepiness Scale (ESS), Pittsburgh Sleep Quality Index (PSQI), and Insomnia Severity Index (ISI).

Results: The North Korean refugees showed more commission errors (CEs, p < 0.05) and a larger standard deviation (SD) of the reaction time $(\mathrm{RT})(\mathrm{p}<0.05)$ in the sustained attention task compared to the South Korean participants. Furthermore, the North Korean refugees showed a shorter period of wake after sleep onset (WASO, $p<0.01$ ), less time spent in N1 ( $p<0.05)$, and more time spent in N2 $(\mathrm{p}<0.05)$. The larger SD of RT in the sustained attention task in the North Korean refugees was positively correlated with WASO $(r=0.62, p<0.01)$ and N1 stage $(r=0.47$, $\mathrm{p}<0.05)$ after controlling for age, sex, BDI, BAI, and IES-R.

Conclusion: The North Korean refugees showed poorer performance on the sustained attention task. Nocturnal PSG revealed shorter WASO and time spent in N1 in this population, which are independently associated with the preservation of attentional capacity. These data suggest that traumatized refugees may compensate for attentional deficits induced by their traumatic experiences via increased sleep continuity.

Keywords: attention, sleep, trauma, North Korean refugees

\section{Introduction}

Before reaching a safe environment, refugees are commonly exposed to psychological trauma in the pre- and post-migration periods. ${ }^{1}$ These traumatic experiences can affect social and psychological functioning, and refugees may also experience stress when adjusting to a new culture. Indeed, the prevalence rates of various psychological problems are reportedly higher in refugees. ${ }^{2}$ North Korean refugees are particularly affected, as many have experienced extremely traumatic experiences such as imprisonment, torture, starvation, witnessing death, human trafficking, and violence. Previous studies have reported that a history of traumatic experiences is a decisive factor in the mental health of North Korean refugees, ${ }^{3-5}$ as well as in their ability to adjust to a new culture. 
Trauma-exposed populations frequently exhibit cognitive dysfunction and sleep disturbances, in addition to other trauma-related symptoms, such as dissociation or negative affect. ${ }^{6,7}$ Refugees are more likely to have had traumatic experiences. Refugees are also at a greater risk of trauma-related sleep problems or cognitive dysfunction. ${ }^{6,8-11}$ Sleep disturbances in refugees are a critical concern, as sleep is more closely correlated with mental health in this population compared to nonrefugee migrant populations. ${ }^{6}$ Indeed, North Korean refugees have been reported to have poor attentional performance and subjective sleep disturbances, both of which are associated with mental health. ${ }^{12-14}$

The influence of sleep on attention is well known. ${ }^{15-17}$ Sleep deprivation impairs cognitive functions including attention, and poor sleep quality has been linked with negative cognitive bias and decreased sustained attention. $^{18}$ Sleep has been proposed to have a bidirectional interaction with cognitive function in traumatized people. ${ }^{19-22}$ However, whether the sleep-attention relationship is enhanced or weakened in traumatized refugees is unclear. Furthermore, since both sleep and attention are strongly affected by depression, anxiety, and other trauma-related symptoms that are very common in refugees, ${ }^{23,24}$ these factors should be considered when investigating the association between attention and sleep in traumatized refugees. ${ }^{7}$

Sleep complaints can be subjective, but may also involve objective physiological changes in sleep architecture. ${ }^{9}$ Whether objective or subjective information about sleep is obtained has implications in clinical and research settings. Subjective measures of sleep, such as questionnaires and interviews, are obviously insufficient to determine objective changes in sleep in traumatized refugees. Overnight polysomnography (PSG) is the most commonly used modality to assess psychological and objective sleep. PSG can provide information about sleep efficiency, sleep stage, arousal, awakening, movement, and respiratory events. Objective sleep has been assessed using PSG in non-refugees. ${ }^{25-30}$ However, no studies have used PSG to investigate sleep in traumatized refugees. Furthermore, no previous studies have investigated the relationship between objective sleep and objective cognitive function in refugees.

Therefore, we investigated the relationship between attentional deficits and objective measures of sleep structure (as measured via PSG) in traumatized North Korean refugees. We developed three hypotheses for the current study. Our first hypothesis was that North Korean refugees would have attentional deficits. Our second hypothesis was that sleep continuity, as measured by PSG, would be disturbed in North Korean refugees compared to South Koreans. Our third hypothesis was that sleep changes, as measured by PSG, would be associated with attentional deficits in traumatized North Korean refugees, even after controlling for other trauma-related symptoms.

\section{Methods}

\section{Participants}

The participants were 32 North Korean refugees (11 males and 21 females; mean age $=33.78 \pm 14.33$ years) and 39 South Korean non-refugees (20 males and 19 females; mean age $=35.03 \pm 11.08$ years). The South Koreans were born and raised in South Korea without any traumatic experiences. The North Korean refugees were born in North Korea or in other countries and ultimately settled in South Korea. Recruiting period of North Korean refugees was from October 2016 to October 2019. Participants were recruited by advertisements with the help of institutions supporting North Korean Refugees. After escaping from North Korea, North Korean refugees usually passed through or stayed over in other countries as China, Russia, Laos, or Thailand before coming to South Korea. The average duration of defection in these third countries was $24.5 \pm 36.4$ months. Mean age of North Korean refugees at the moment of escape from North Korea was 24.5 \pm 10.7 years old. All participants have separation experience from family or close friends, $60 \%$ of participants have remaining family members in North Korea. The averaged settlement period in South Korea was 88.9 50.7 months.

Inclusion criteria for the North Korean refugees for the current study were as follows: (1) born in North Korea or during defection from North Korea, (2) more than 15 years old, (3) settlement in South Korea after 2000, (4) traumatic experience in North Korean or defection periods. All the North Korean refugees reported having traumatic experiences before settling in South Korea, such as neglect from caregivers, witness of public execution or death of a family member, or near-death starvation. The exclusion criteria were as follows: (1) aged $<18$ or $>60$ years, (2) repeated or recent seizures, brain injury or organic brain syndrome, (3) severe personality disorder, (4) intellectual disability with a Full Scale IQ (FSIQ) score under 70, (5) emergent psychiatric state (serious suicidality or fullblown psychotic/delirious features), (6) employment as 
a shift worker, (7) severe sleep apnea [Apnea Hypopnea Index (AHI) >30] according to PSG data or previous evaluation history, (8) severe limb movement during sleep [Periodic Limb Movement Index (PLMI) >50]. All procedures in the current study were conducted in accordance with the ethical standards of the relevant national and institutional committees on human experimentation and the Helsinki Declaration of 1964, as revised in 2013. The study protocol involving human subjects was approved by the Institutional Review Board of Samsung Medical Center (approval number: 2016-07-049). Informed written consent was provided by all participants.

\section{Assessment of Psychological Symptoms}

We used the Beck Depression Inventory (BDI) to measure the severity of depressive symptoms. ${ }^{31}$ The BDI includes 21 items and covers various domains of depressive symptoms. Each item is scored from 0 to 3. Higher total scores represent more severe depression. We used the Beck Anxiety Inventory (BAI) to measure general anxiety symptoms. ${ }^{32,33}$ The BAI, which has 21 items, is a selfrated scale designed to assess the severity of anxiety symptoms in adults. Korean versions of BDI and BAI (from Korea Psychology Co., LTD.) were used for the current study. ${ }^{34,35}$

We used the Clinician-Administered PTSD Scale (CAPS) to diagnose PTSD in the North Korean refugees. ${ }^{36}$ Clinician interviews of the North Korean participants were based on 30 items pertaining to PTSD symptoms, such as re-experiencing, avoidance, numbing, and hyperarousal. We used the Impact of Event ScaleRevised (IES-R) to assess PTSD symptoms. ${ }^{37,38}$ The IES-R comprises 22 items and covers topics including intrusive re-experiencing of symptoms, avoidance, and hyperarousal. The IES-R and CAPS were only conducted for the North Korean refugees, as the South Korean participants had no history of traumatic experiences.

\section{Subjective Assessment of Sleep Quality}

We used the Pittsburgh Sleep Quality Index (PSQI), which includes 18 items related to sleep structure and patterns, to subjectively assess sleep quality over the last 1 month. The index has seven components: subjective sleep quality, sleep latency, sleep duration, habitual sleep efficiency, sleep disturbance, use of sleep medication, and daytime dysfunction. ${ }^{39}$ Higher total scores indicate poorer sleep. ${ }^{40}$ We used the Epworth Sleepiness Scale (ESS), which is a four-point scale describing eight situations, to measure daytime sleepiness. ${ }^{41}$ Korean versions of ESS, which had been validated by Sleep Center of Keimyung University Dongsan Medical Center, were used for the current study. ${ }^{42}$ We used the Insomnia Severity Index (ISI) to examine initial, maintenance, and terminal insomnia (3 items) and satisfaction, interference, noticeability, and distress related to sleep problems (4 items). ${ }^{43}$ Korean versions of ISS, which had been validated by Sleep Center of Keimyung University Dongsan Medical Center, were used for the current study. ${ }^{44}$

\section{Computerized Attention Test}

We assessed sustained and divided attention in all participants using the computerized Comprehensive Attention Test (CAT). ${ }^{45}$ CAT-pro produced by Happymind INC. was used as the software for the current study. The CAT, which comprises visual and auditory continuous performance tests, comprehensively evaluates attention and working memory. We used the CAT to test for attention deficits in both the North Korean refugees and South Korean participants. CAT was conducted immediately before conducting PSG for all participants.

The sustained attention task assesses the ability of an individual to maintain a consistent behavioral response during exposure to continuous and repetitive stimuli. There are four different types of visual stimuli (cross, diamond, oval, and X-shape) used in the sustained attention task. The visual stimuli are presented randomly on the computer screen every 2 seconds during 10 minutes. The participants are asked to respond to every stimulus except the X-shape. Thus, the task measures response inhibition to certain visual stimuli during sustained attention. As the proportion of each visual type is set to the same, which means that there are $75 \mathrm{X}$-shape stimuli (25\%) among total 300 stimuli. The divided attention task assesses the ability to process different kinds of sensory stimuli simultaneously. Visual stimuli and auditory stimuli are presented simultaneously on screen every 2 seconds for 3 minutes and 20 seconds. Three types of auditory stimuli (beep, bell, and click) and three types of visual stimuli (triangle, circle, and rectangle) are combined and presented randomly. Participants were asked to respond when either auditory or visual stimuli were the same as the previous stimuli. The correct response ratio is set to be $50 \%$, as 50 stimuli among 100 stimuli are the same as the previous stimuli either in auditory or visual characteristics. After verbal explanation, every participant performed 10 trials before the beginning of the actual test. 
We measured the omission error (OE) and commission error (CE) rates in each attention task. An OE is defined as a missed response to a target stimulus, and thus represents inattention. $\mathrm{A} \mathrm{CE}$ is defined as impulsive response to a non-target stimulus, and thus represents impulsivity. We also calculated the reaction time (RT) and standard deviation (SD) of the RT for correct responses in each attention task. The SD of RT indicates the ability to maintain the same level of attention for a certain period of time, as shown in previous studies. ${ }^{46-48}$

\section{Polysomnography}

Overnight PSG to objectively assess sleep structure was conducted for one night. PSG procedure was conducted according to the American Academy of Sleep Medicine (AASM) Guideline for Type I sleep study. ${ }^{49}$ PSG device was N7000 PSG with a 32 channel head unit produced by Embla. PSG data were recorded and processed with the software Remlogic version 3.4.

The sleep variables in the present study included total sleep time (TST), wake after sleep onset (WASO), percentage of time spent in each sleep stage (N1-N3) and in rapid eye movement (REM) sleep, sleep latency, slow wave sleep (SWS) latency, and the total arousal index. WASO is defined as the wake time in minutes during the period after sleep onset. The total arousal index was defined as the number of EEG arousals per hour of sleep. PSG data were also used to screen for sleep disorders. Two trained polysomnographic technologists scored all the PSG data according to the American Academy of Sleep Medicine (AASM) Manual for the Scoring of Sleep and Associated Events, version 2.2. ${ }^{50}$

\section{Statistical Analysis}

All statistical analyses were performed using SPSS statistics software (ver. 25.0; SPSS Inc., Chicago, IL, USA). Regarding continuous variables, differences in demographic and clinical characteristics between the North Korean refugees and South Korean participants were evaluated using $t$-tests. Differences between the two groups of categorical variables were evaluated using the chi-square test. Pearson's correlation analysis was used to evaluate the association between attentional deficits (ie, CAT data) and sleep architecture (ie, PSG variables). A partial correlation analysis was performed, adjusting for age, sex, BDI score, and BAI score. For investigating the interaction effects of refugee status, regression analysis (independent variable: CAT, dependent variables: PSG, refugee status, and PSG $\mathrm{X}$ refugee status interaction) was additionally conducted for variables showing significant correlation between sleep and attention in North Korean refugees. The level of statistical significance was set at $P<0.05$.

\section{Results}

\section{Demographic and Clinical Characteristics}

Table 1 presents the demographic and clinical characteristics of both the North Korean refugees and South Korean participants. No difference in age or sex ratio was

Table I Demographic and Clinical Characteristics of North Korean Refugees and South Koreans

\begin{tabular}{|c|c|c|c|c|}
\hline & North Korean Refugees $(n=32)$ & South Korean $(n=39)$ & $\mathbf{t}$ & P value \\
\hline & Mean \pm SD & Mean \pm SD & & \\
\hline Age & $33.78 \pm 14.33$ & $35.03 \pm 11.08$ & $\mathrm{t}=\mathrm{I} .43$ & 0.68 \\
\hline Female, No.(\%) & $21(65.6 \%)$ & 19(48.7\%) & $\chi^{2}=2.0$ & 0.15 \\
\hline BDI & $13.59 \pm 8.24$ & $9.36 \pm 9.70$ & $\mathrm{t}=1.96$ & 0.05 \\
\hline BAI* & $16.41 \pm 9.43$ & $9.54 \pm 11.85$ & $\mathrm{t}=2.66$ & 0.01 \\
\hline CAPS, No.(\%) & $16(50 \%)$ & & & \\
\hline IES (sum) & $20 \pm 20.84$ & & & \\
\hline IES reexperience & $7.06 \pm 7.52$ & & & \\
\hline IES avoidance & $7.94 \pm 8.51$ & & & \\
\hline IES hyperarousal & $5.00 \pm 5.64$ & & & \\
\hline PSQI & $8.53 \pm 3.74$ & $8.08 \pm 4.59$ & $\mathrm{t}=0.45$ & 0.65 \\
\hline ISI & $11.50 \pm 7.11$ & II. $.38 \pm 8.40$ & $\mathrm{t}=0.06$ & 0.95 \\
\hline ESS & $8.28 \pm 3.81$ & $6.74 \pm 3.65$ & $t=1.73$ & 0.09 \\
\hline
\end{tabular}

Note: *p value $<0.05$.

Abbreviations: BDI, Beck Depression Inventory; BAI, Beck Anxiety Inventory; IES, Impact of Event Scale-Revised; CAPS, Clinician-administered PTSD Scale; ESS, Epworth Sleepiness Scale; PSQI, Pittsburgh Sleep Quality Index; ISI, Insomnia Severity Index. 
observed between the two groups. The BAI score in the North Korean refugees was significantly higher than that in the South Korean participants $(\mathrm{t}=2.66, \mathrm{p}<0.05)$. BDI, PSQI, ISI, and ESS scores were not significantly different between the two groups.

\section{Attention Deficits in North Korean Refugees and South Korean Participants}

The North Korean refugees had a higher CE rate (17.0 \pm 26.0 vs $6.9 \pm 6.7, \mathrm{p}<0.05)$ and a larger SD of RT $(107.6 \pm$ 49.5 vs $84.3 \pm 27.9, \mathrm{p}<0.05)$ in the sustained attention task compared to the South Korean participants (Table 2). Performance on the divided attention task was not significantly different between the groups. The significant differences between CE and SD of RT on sustained attention between North Korean refugees and South Koreans remain significant after controlling sex.

For each group, we examined the correlation between attentional deficits and psychiatric/sleep complaints, after controlling for age and sex. For North Korean refugees, a higher BDI was significantly associated with a longer RT in the divided attention task $(\mathrm{r}=0.44, \mathrm{p}<0.05)$. Furthermore, PSQI scores were positively associated with the OE rate $(\mathrm{r}=0.40, \mathrm{p}<0.05)$, RT $(\mathrm{r}=0.40, \mathrm{p}<0.05)$ and SD of RT $(r=0.40, p<0.05)$ in the divided attention

Table 2 Attentional Performances of North Korean Refugees and South Koreans

\begin{tabular}{|l|c|c|c|c|}
\hline & $\begin{array}{c}\text { North } \\
\text { Korean } \\
\text { Refugees } \\
\text { (n=32) }\end{array}$ & $\begin{array}{c}\text { South } \\
\text { Korean } \\
(\mathbf{n = 3 9 )}\end{array}$ & t & P value \\
\cline { 2 - 3 } & Mean \pm SD & $\begin{array}{c}\text { Mean } \pm \\
\text { SD }\end{array}$ & \\
\hline Sustained attention \\
\hline OE (No.) & $15.9 \pm 45.4$ & $8.8 \pm 37.5$ & 0.73 & 0.47 \\
CE (No.) & $17.0 \pm 26.0$ & $6.9 \pm 6.7$ & 2.14 & 0.04 \\
RT (ms) & $419.8 \pm 146.9$ & $406.2 \pm 49.5$ & 0.46 & 0.65 \\
SD of RT* & $107.6 \pm 49.5$ & $84.3 \pm 27.9$ & 2.50 & 0.02 \\
\hline Divided attention & \multicolumn{3}{|c}{} \\
\hline OE (No.) & $10.2 \pm 7.2$ & $10.1 \pm 11.4$ & 0.02 & 0.98 \\
CE (No.) & $6.0 \pm 8.0$ & $5.4 \pm 7.0$ & 0.35 & 0.73 \\
RT (ms) & $718.0 \pm 226.8$ & $755.6 \pm 181.0$ & 0.78 & 0.44 \\
SD of RT & $232.7 \pm 81.0$ & $214.3 \pm 78.2$ & 0.96 & 0.34 \\
\hline
\end{tabular}

Note: ${ }^{*} \mathrm{p}$ value $<0.05$

Abbreviations: $\mathrm{OE}$, omission error; $\mathrm{CE}$, commission error; RT, mean reaction time to correct responses; SD of RT, standard deviation of reaction time to correct responses. task. Among the South Korean participants, a higher BDI score was significantly associated with a higher OE rate in the divided attention task $(r=0.44, p<0.05)$. No significant correlations were observed between any other psychiatric/sleep complaints and CAT variables in the South Korean participants.

\section{Sleep Architecture in the North Korean Refugees and South Korean Participants}

In the overnight PSG data, the North Korean refugees had a shorter WASO duration $(26.0 \pm 24.5$ vs $46.9 \pm 33.1 \mathrm{~min}$, $\mathrm{p}<0.01)$, lower amount of time spent in N1 (11.1 \pm 7.1 vs $15.1 \pm 8.9 \%, \mathrm{p}<0.05)$, and higher amount of time spent in $\mathrm{N} 2(58.2 \pm 7.4$ vs $54.0 \pm 7.6 \%, \mathrm{p}<0.05)$ compared to the South Korean participants (Table 3). The other PSG variables were not significantly different between the two groups. The significant differences of WASO, N1 and N2 between North Korean refugees and South Koreans remain significant after controlling sex.

We examined the correlation between PSG findings and psychiatric/sleep complaints in each group, controlling for age and sex. Among the North Korean refugees, we found a significant association between the BAI score and sleep latency $(\mathrm{r}=0.48, \mathrm{p}<0.01)$. Furthermore, the ESS score was negatively associated with $\mathrm{N} 1$ stage $(\mathrm{r}=-0.50$, $\mathrm{p}<0.01)$ and REM latency $(\mathrm{r}=-0.42, \mathrm{p}<0.05)$, and positively associated with REM stage $(r=0.42, p<0.05)$. For South Korean participants, the PSQI score was positively associated with SWS latency $(r=0.40, p<0.05)$.

\section{Impact of Sleep Architecture on Attention}

Figure 1 shows the association between the PSG data and performance in the attention tasks for each group, controlling for age, sex, BDI score, and BAI score. In the North Korean refugees, a higher $\mathrm{OE}$ in the sustained attention task was correlated with a lower amount of N2 $(r=-0.47, \mathrm{p}<$ $0.05)$ and a higher amount of $\mathrm{N} 3(\mathrm{r}=0.42, \mathrm{p}<0.05)$. More CEs in the sustained attention task were significantly associated with a higher total arousal index $(\mathrm{r}=0.47, \mathrm{p}<0.05)$. Furthermore, a larger SD of RT in the sustained attention task was correlated with a longer WASO duration $(\mathrm{r}=0.62$, $\mathrm{p}<0.01)$, higher amount of N1 ( $\mathrm{r}=0.47, \mathrm{p}<0.05)$, lower amount of N3, and longer SWS latency $(r=0.69, \mathrm{p}<0.01)$ (Figure 2). The association remained significant after removing an outlier in Figure 2. In terms of divided attention in the North Korean refugees, a higher OE rate was correlated with 
Table 3 Polysomnography (PSG) Data of North Korean Refugees and South Koreans

\begin{tabular}{|c|c|c|c|c|}
\hline \multirow[t]{2}{*}{ PSG Parameter } & $\begin{array}{c}\text { North Korean Refugees } \\
\qquad(n=32)\end{array}$ & South Korean $(n=39)$ & \multirow[t]{2}{*}{$\mathbf{t}$} & \multirow[t]{2}{*}{$P$ value } \\
\hline & Mean \pm SD & Mean \pm SD & & \\
\hline Total sleep time (min) & $403.5 \pm 72.7$ & $409.5 \pm 45.5$ & -0.43 & 0.67 \\
\hline Sleep efficiency (\%) & $89.6 \pm 11.9$ & $86.6 \pm 8.6$ & 1.22 & 0.23 \\
\hline WASO $(\mathrm{min})^{* *}$ & $26.0 \pm 24.5$ & $46.9 \pm 33.1$ & -3.06 & 0.003 \\
\hline NI (\%)* & $\mid I . I \pm 7.1$ & $15.1 \pm 8.9$ & -2.05 & 0.04 \\
\hline N2 $(\%)^{*}$ & $58.2 \pm 7.4$ & $54.0 \pm 7.6$ & 2.29 & 0.03 \\
\hline N3 (\%) & $9.3 \pm 7.9$ & $10.7 \pm 9.4$ & -0.70 & 0.49 \\
\hline REM (\%) & $21.4 \pm 5.4$ & $25.2 \pm 28.4$ & -0.74 & 0.46 \\
\hline Sleep latency (min) & $16.5 \pm 37.5$ & $15.7 \pm 17.0$ & 0.11 & 0.91 \\
\hline SWS latency (min) & $27.2 \pm 21.8$ & $35.0 \pm 47.3$ & -0.84 & $0.4 I$ \\
\hline REM latency (min) & $108.5 \pm 65.2$ & $113.8 \pm 49.5$ & -0.38 & 0.70 \\
\hline Total arousal index (min) & $2.5 \pm 2.5$ & $3.2 \pm 4.3$ & -0.90 & 0.37 \\
\hline
\end{tabular}

Notes: ${ }^{*} p$ value $<0.05$. ${ }^{* *} p$ value $<0.01$.

Abbreviations: WASO, wake after sleep onset; SWS, slow wave sleep; REM, rapid eye movement.

a shorter TST $(\mathrm{r}=-0.59, \mathrm{p}<0.01)$, as well as a decreased sleep efficiency $(r=-0.49, p<0.05)$. The SD of RT for the divided attention task was significantly correlated with the TST $(r=-0.43, p<0.05)$. Even after controlling for the IES$\mathrm{R}$ score as an additional covariate in North Korean refugees, similar results were obtained.

In terms of sustained attention in the South Korean participants, we found a positive correlation between the OE rate and WASO $(r=0.42, p<0.05)$ and between the $\mathrm{SD}$ of RT and sleep latency $(\mathrm{r}=0.47, \mathrm{p}<0.01)$ after controlling for age, sex, BDI score, and BAI score. In the divided attention task, a higher $\mathrm{CE}$ rate was correlated with a shorter TST $(r=-0.43, \mathrm{p}<0.05)$ and a longer SWS latency $(r=0.45, \mathrm{p}<0.05)$ in the South Korean participants. Furthermore, a larger SD of RT in the divided attention task was correlated with lower sleep efficiency $(\mathrm{r}=-0.36, \mathrm{p}<0.05)$ in this population.

Linear regression model showed significant interaction effects by refugee status on the association between the sustained attention and the polysomnographic findings in total subjects. There were significant interaction effects by refugees status on the relationships between $\mathrm{OE}$ and $\mathrm{N} 2(\mathrm{~B}=-2.89$, $\mathrm{p}<0.05), \mathrm{OE}$ and N3 ( $\mathrm{B}=1.783, \mathrm{p}<0.05), \mathrm{CE}$ and $\mathrm{AI}(\mathrm{B}=$ 4.398, $p<0.01)$, SD of RT and WASO $(B=0.934, p<0.01)$, SD of RT and N3 (B = -3.144, p $<0.01)$, and SD of RT and SWS latency $(B=0.992, p<0.01)$. Only the relationship between $S D$ of RT and N1 was not significantly affected by interaction effects by refugee status. On the contrary, there were no interaction effects by refugee status on the association between the divided attention and the polysomnographic findings.

\section{Discussion}

Compared to the South Korean participants, the North Korean refugees in the present study showed poorer performance in the attention task, as well as lower levels of arousal and awakening during PSG. However, poor performance in the attention task of North Korean refugees was correlated with higher levels of arousal and more frequent awakening during nocturnal sleep. Taken together, these findings suggest that reduced arousal and awakening during sleep in the refugee group might affect attentional deficits after traumatic experiences. To the best of our knowledge, this study is the first to investigate the association between objective sleep measurements (ie, PSG data) and cognitive function in refugees.

The decreased attentional performance of North Korean refugees supported our first hypothesis. The higher $\mathrm{CE}$ and larger SD of RT in the sustained attention task of North Korean refugees may reflect increased cognitive impulsivity, as well as difficulty maintaining attention for a certain period of time. Our findings are consistent with previous studies reporting attentional deficits in North Korean refugees and other refugee populations. ${ }^{8}$

In contrast to our second hypothesis, the North Korean refugees showed shorter WASO periods (ie less awakening), as well as less time spent in stage $\mathrm{N} 1$ and more time spent in stage N2 (ie, they easily transitioned to stage N2 without interruption of N1 sleep) during overnight PSG. These findings indicate that traumatized North Korean refugees had good sleep continuity. This finding is in contrast to previous studies reporting poorer sleep quality 
in traumatized people. ${ }^{51,52}$ This discrepancy may be due to differences between traumatized North Korean refugees and other traumatized populations. Methodological differences may also explain this finding; while we used PSG, many previous studies used subjective sleep questionnaires.

Our finding that attentional performance in North Korean refugees was associated with objective sleep supported our final hypothesis. Performance on sustained attention tasks in North Korean refugees was associated with qualitative sleep variables, such as arousal and awakening, and increased N2 sleep. Furthermore, performance on the divided attention task in North Korean refugees was associated with quantitative sleep variables, such as TST and sleep efficiency. This finding suggests that improving qualitative and quantitative sleep could reduce attention deficits in North Korean refugees. Furthermore, this finding is consistent with previous studies reporting an association between decreased sleep continuity and cognitive decline. ${ }^{21,53-57}$ The association between sleep and trauma-related symptoms has been widely studied, ${ }^{58,59}$ and REM sleep and SWS have been found to play a role in altered cognitive performance in traumatized people. ${ }^{59-61}$ Furthermore, a relationship has been established between reduced arousal during sleep and adaptive cognitive processing of traumatic

Partial correlation coefficient

\begin{tabular}{llllllll}
\hline-1 & -0.75 & -0.5 & -0.25 & 0 & 0.25 & 0.5 & 0.75
\end{tabular}

\begin{tabular}{|c|c|c|c|c|c|c|c|c|}
\hline & \multicolumn{4}{|c|}{ Sustained attention } & \multicolumn{4}{|c|}{ Divided attention } \\
\hline & OE & CE & RT & SD of RT & OE & CE & RT & SD of RT \\
\hline TST & -0.09 & 0.22 & -0.19 & -0.19 & $-0.57^{* *}$ & 0.10 & -0.33 & $-0.40^{*}$ \\
\hline Sleep efficiency & -0.04 & 0.11 & -0.21 & -0.27 & $-0.47^{*}$ & 0.04 & -0.21 & -0.19 \\
\hline WASO & 0.12 & 0.17 & 0.16 & $0.59^{* *}$ & 0.32 & 0.18 & -0.12 & 0.24 \\
\hline N1 & 0.12 & 0.16 & 0.06 & $0.41^{*}$ & 0.27 & 0.21 & -0.10 & 0.15 \\
\hline N2 & $-0.46^{*}$ & 0.09 & 0.14 & 0.12 & -0.18 & 0.01 & -0.14 & -0.30 \\
\hline N3 & $0.41^{\star}$ & -0.09 & -0.31 & $-0.48^{*}$ & 0.03 & -0.11 & 0.31 & 0.11 \\
\hline REM & -0.17 & -0.19 & 0.17 & -0.01 & -0.17 & -0.13 & -0.13 & 0.01 \\
\hline Sleep latency & 0 & -0.19 & 0.1 & -0.10 & 0.23 & -0.13 & 0.26 & 0 \\
\hline SWS latency & 0.02 & 0.19 & 0.18 & $0.68^{* *}$ & 0.21 & 0.31 & -0.12 & 0.06 \\
\hline REM latency & 0.05 & 0 & 0.06 & 0.05 & 0.12 & 0.1 & 0.23 & 0.27 \\
\hline Total arousal index & 0.24 & $0.47^{*}$ & -0.29 & 0.25 & 0.23 & -0.01 & -0.34 & -0.14 \\
\hline
\end{tabular}

A North Korean refugees

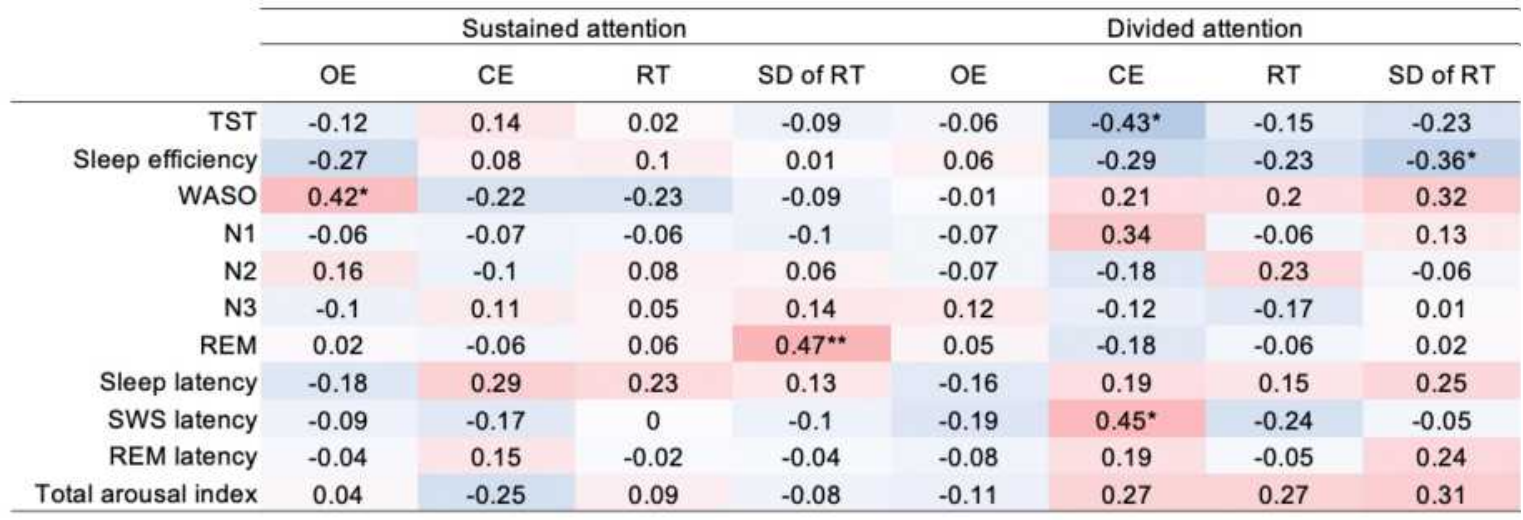

\section{B South Korean}

Figure I Association between attention deficits and objective sleep architecture in traumatized North Korean refugees. Significant correlation between the PSG data and performance in the attention tasks was found in each group, the North Korean refugees (A) and the South Koreans (B).

Notes: ${ }^{*} \mathrm{p}$ value $<0.005$, **p value $<0.01$, controlled variables: age, sex, BDI, BAI.

Abbreviations: OE, omission error; CE, commission error; RT, mean reaction time to correct responses; SD, standard deviation; WASO, wake after sleep onset; SWS slow wave sleep; REM, rapid eye movement. 


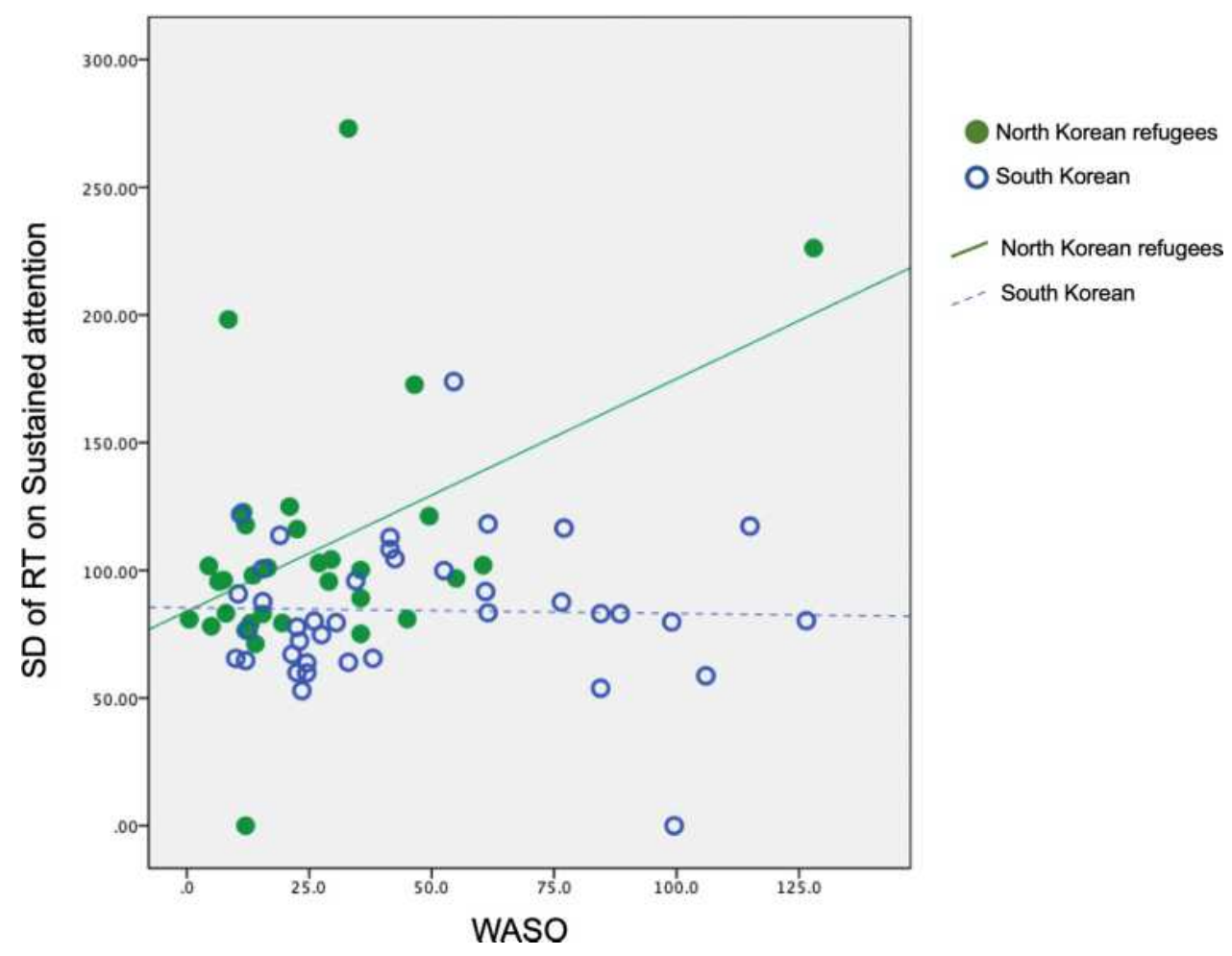

Figure 2 The association of SD of RT on sustained attention with WASO.

Abbreviations: SD of RT, standard deviation of mean reaction time of correct response; WASO, wake after sleep onset.

experiences. ${ }^{62,63}$ Sleep spindles, as well as K-complexes and slow waves (markers of N2 and N3, respectively), have been implicated in memory consolidation and activity in the thalamo-cortical network. ${ }^{64}$ These variables have been found to be altered in North Korean refugees. Reduced N3 and increased REM density have also been associated with a poorer prognosis after traumatic experiences. $^{63,65}$

While our North Korean refugees exhibited attentional deficits, objective sleep continuity in this group was less disturbed than that in the South Korean participants. However, poorer attentional performance in the North Korean refugees was associated with "disturbed" sleep but not "better" sleep continuity. These associations were also more prominent in North Korean refugees than in South Korean participants. Therefore, the increased sleep continuity observed in traumatized North Korean refugees might play a role in the preservation of attention. We speculate that increased sleep continuity in refugees might represent a compensatory physiological response to traumatic events promoting the adaptation necessary for survival. ${ }^{66}$

Although the mechanism via which altered sleep might compensate for attentional deficits in traumatized refugees is unclear, the association between sleep and attention in this population may be related to changes in noradrenergic activity. Aberrant firing and signaling in the locus coeruleus after traumatic experiences may induce both sleep abnormalities and cognitive dysfunction via activation of the noradrenergic system, which controls activity in the hippocampus, prefrontal cortex, and amygdala. ${ }^{63}$ Indeed, the activation of arousal systems by noradrenaline is implicated in the development and maintenance of sleep disturbances. $^{22}$

Another probable explanation for lower levels of arousal and awakening in North Korean refugees would be sleep deprivation potentially induced by socioeconomic difficulties of North Korean refugees. WASO and N1 usually decrease after sleep deprivation. The negative association between daytime sleepiness and $\mathrm{N} 1$ in the current study supports this possibility. However, the association between the higher attentional capacity and the lower arousal (more sleep deprived status) did not support the notion.

As mentioned above, sleep and attention can have a synergistic effect on psychiatric symptoms in traumatized people, as they are both essential features of traumarelated psychiatric disorders. The current data show an 
association between attention and objective sleep, independent of age, sex, depression, anxiety, and other traumarelated symptoms. Thus, our findings suggest that attentional deficits in traumatized refugees might be directly affected by sleep itself, rather than indirectly via other trauma-related emotional disturbances.

Interestingly, refugee status affects the relationship between selective attention and sleep, while the relationship between divided attention and sleep is not affected by the refugee status. This result suggests that the association between decreased arousal during sleep and enhanced selective attention would be more noticeable for North Korean refugees, while the association between total sleep time and enhanced divided attention was not. Although it is unclear why the sleep-attention relationship of refugees is more prominent in selective attention domain rather than in divided attention, it may be associated with severe impairments of selective attention or inhibitory control in traumatized people reported in previous studies. ${ }^{67,68}$

Our findings have several clinical and research implications. The association between sleep and attention may provide clues regarding potential therapeutic targets for treating traumatized refugees. Indeed, a previous study reported that interventions targeting sleep in trauma victims could improve other trauma-related symptoms. ${ }^{69}$ Our findings suggest that attentional deficits can also be addressed by improving sleep quality in refugees. Future studies based on the current findings should investigate the relationship between sleep and other cognitive domains in this population, and use brain imaging to determine the neurobiological pathway underlying the associations among sleep, cognition, and trauma.

\section{Limitations}

This study has several limitations. First, the sample size was small. However, this is the first study to use PSG in a refugee population. Second, as the study was crosssectional, we could not investigate the sequential effects of traumatic experiences, nor establish causal relationships among sleep, cognition, and trauma. Third, the control group consisted only of non-traumatized South Korean individuals. However, the recruitment of nontraumatized North Koreans is practically impossible, as North Korea is a completely isolated society with very little contact with the rest of the world. Single night PSG without adaptive nights was another limitation of the current study. One previous study reported that about $50 \%$ of patients showed first night effect, while about
$20 \%$ of patients showed reverse first night effects. ${ }^{70}$ Therefore, first night effects may affect our PSG results, although the first night effect can be partially offset by the reverse first night effect.

\section{Conclusion}

Using PSG, we found a negative correlation between attentional deficits and sleep continuity in North Korean refugees. Our data suggest that increased sleep continuity in traumatized refugees may compensate for attentional deficits induced by a history of traumatic experiences.

\section{Acknowledgments}

This research was supported by the Brain Research Program through the National Research Foundation of Korea (NRF), funded by the Korean government (MSIT) (No. 2016M3C7A1904338), National Research Foundation of Korea (NRF) grant funded by the Korean government (MSIT) (No. 2020R1F1A1049200, No. 2016R1A2B4011561), and the Bio \& Medical Technology Development Program of the National Research Foundation (NRF) funded by the Korean government (MSIT) (No. 2020M3E5D9080561).

\section{Disclosure}

The authors report no conflicts of interest in this work.

\section{References}

1. Steel Z, Chey T, Silove D, Marnane C, Bryant RA, van Ommeren M. Association of torture and other potentially traumatic events with mental health outcomes among populations exposed to mass conflict and displacement: a systematic review and meta-analysis. JAMA. 2009;302(5):537-549. doi:10.1001/jama.2009.1132

2. Turrini G, Purgato M, Ballette F, Nosè M, Ostuzzi G, Barbui C. Common mental disorders in asylum seekers and refugees: umbrella review of prevalence and intervention studies. Int J Ment Health Syst. 2017;11(1):51. doi:10.1186/s13033-017-0156-0

3. Hong CH, Jeon WT, Lee CH, Kim DK, Han M, Min SK. Relationship between traumatic events and posttraumatic stress disorder among North Korean refugees. J Korean Neuropsychiatr Assoc. 2005;44 (6):714-720.

4. Jeon W-T, Yu S-E, Cho Y-A, Eom J-S. Traumatic experiences and mental health of north Korean refugees in South Korea. Psychiatry Investig. 2008;5(4):213-220. doi:10.4306/pi.2008.5.4.213

5. Lee KE, Moon CS, An JH, et al. Prevalence of DSM-IV major psychiatric disorders among North Korean defectors in South Korea. Psychiatry Investig. 2020;17(6):541. doi:10.30773/pi.2019.0217

6. Richter K, Baumgärtner L, Niklewski G, et al. Sleep disorders in migrants and refugees: a systematic review with implications for personalized medical approach. EPMA J. 2020;11:251-260. doi:10.1007/s13167-020-00205-2

7. Sandahl H, Jennum P, Baandrup L, Poschmann IS, Carlsson J. Treatment of sleep disturbances in trauma-affected refugees: study protocol for a randomised controlled trial. Trials. 2017;18(1):1-13. doi:10.1186/s13063-017-2260-5 
8. Lee YJ, Jun JY, Park J, et al. Effects of psychiatric symptoms on attention in North Korean refugees. Psychiatry Investig. 2016;13 (5):480. doi:10.4306/pi.2016.13.5.480

9. Sandahl H, Vindbjerg E, Carlsson J. Treatment of sleep disturbances in refugees suffering from post-traumatic stress disorder. Transcult Psychiatry. 2017;54(5-6):806-823. doi:10.1177/1363461517746314

10. Mirabolfathi V, Schweizer S, Moradi A, Jobson L. Affective working memory capacity in refugee adolescents. Psychol Trauma Theory Res Pract Policy. 2020. doi:10.1037/tra0000552

11. Ainamani HE, Elbert T, Olema DK, Hecker T. PTSD symptom severity relates to cognitive and psycho-social dysfunctioning-a study with Congolese refugees in Uganda. Eur J Psychotraumatol. 2017;8(1):1283086. doi:10.1080/20008198.2017.1283086

12. Kim HH, Lee YJ, Kim HK, et al. Prevalence and correlates of psychiatric symptoms in North Korean defectors. Psychiatry Investig. 2011;8(3):179. doi:10.4306/pi.2011.8.3.179

13. Lee Y-JG, Jun JY, Lee YJ, et al. Insomnia in North Korean refugees: association with depression and post-traumatic stress symptoms. Psychiatry Investig. 2016;13(1):67. doi:10.4306/pi.2016.13.1.67

14. Mysliwiec V, O’Reilly B, Polchinski J, Kwon HP, Germain A, Roth BJ. Trauma associated sleep disorder: a proposed parasomnia encompassing disruptive nocturnal behaviors, nightmares, and REM without atonia in trauma survivors. J Clin Sleep Med. 2014;10 (10):1143-1148. doi: $10.5664 /$ jcsm. 4120

15. Fulda S, Schulz H. Cognitive dysfunction in sleep disorders. Sleep Med Rev. 2001;5(6):423-445. doi:10.1053/smrv.2001.0157

16. Kim S, Park J, Min Oh S, et al. Correlates of performances on computerized attention tasks in patients with insomnia disorder. Chronobiol Med. 2020;2(1):25-31. doi:10.33069/cim.2019.0031

17. Kobayashi I, Mellman TA, Altaee D, Howell MK, Lavela J. Sleep and processing of trauma memories. J Trauma Stress. 2016;29 (6):568-571. doi:10.1002/jts.22137

18. Gobin CM, Banks JB, Fins AI, Tartar JL. Poor sleep quality is associated with a negative cognitive bias and decreased sustained attention. J Sleep Res. 2015;24(5):535-542. doi:10.1111/jsr.12302

19. Brock MS, Powell TA, Creamer JL, Moore BA, Mysliwiec V. Trauma associated sleep disorder: clinical developments 5 years after discovery. Curr Psychiatry Rep. 2019;21(9):1-11. doi:10.1007/ s11920-019-1066-4

20. Lamond N, Dawson D. Quantifying the performance impairment associated with fatigue. $J$ Sleep Res. 1999;8(4):255-262. doi:10.1046/j.1365-2869.1999.00167.x

21. Lowe CJ, Safati A, Hall PA. The neurocognitive consequences of sleep restriction: a meta-analytic review. Neurosci Biobehav Rev. 2017;80:586-604. doi:10.1016/j.neubiorev.2017.07.010

22. Sinha SS. Trauma-induced insomnia: a novel model for trauma and sleep research. Sleep Med Rev. 2016;25:74-83. doi:10.1016/j. smrv.2015.01.008

23. Alvaro PK, Roberts RM, Harris JK. A systematic review assessing bidirectionality between sleep disturbances, anxiety, and depression. Sleep. 2013;36(7):1059-1068. doi:10.5665/sleep.2810

24. Sunderajan P, Gaynes BN, Wisniewski SR, et al. Insomnia in patients with depression: a STAR* D report. CNS Spectr. 2010;15 (6):394-404. doi:10.1017/S1092852900029266

25. Alfano CA, Reynolds K, Scott N, Dahl RE, Mellman TA. Polysomnographic sleep patterns of non-depressed, non-medicated children with generalized anxiety disorder. $J$ Affect Disord. 2013;147(1-3):379-384. doi:10.1016/j.jad.2012.08.015

26. Baglioni C, Nanovska S, Regen W, et al. Sleep and mental disorders: a meta-analysis of polysomnographic research. Psychol Bull. 2016;142(9):969. doi:10.1037/bul0000053

27. Breslau N, Roth T, Burduvali E, Kapke A, Schultz L, Roehrs T. Sleep in lifetime posttraumatic stress disorder: a community-based polysomnographic study. Arch Gen Psychiatry. 2004;61(5):508-516. doi:10.1001/archpsyc.61.5.508
28. Castro LS, Castro J, Hoexter MQ, et al. Depressive symptoms and sleep: a population-based polysomnographic study. Psychiatry Res. 2013;210(3):906-912. doi:10.1016/j.psychres.2013.08.036

29. Fuller KH, Waters WF, Binks PG, Anderson T. Generalized anxiety and sleep architecture: a polysomnographic investigation. Sleep. 1997;20(5):370-376. doi:10.1093/sleep/20.5.370

30. Horváth A, Montana X, Lanquart J-P, et al. Effects of state and trait anxiety on sleep structure: a polysomnographic study in 1083 subjects. Psychiatry Res. 2016;244:279-283. doi:10.1016/j.psychres.2016.03.001

31. Beck AT, Ward CH, Mendelson M, Mock J, Erbaugh J. An inventory for measuring depression. Arch Gen Psychiatry. 1961;4(6):561-571. doi:10.1001/archpsyc.1961.01710120031004

32. Beck AT, Epstein N, Brown G, Steer RA. An inventory for measuring clinical anxiety: psychometric properties. J Consult Clin Psychol. 1988;56(6):893. doi:10.1037/0022-006X.56.6.893

33. Yook SP, Kim ZS. A clinical study on the Korean version of Beck Anxiety Inventory: comparative study of patient and non-patient. Korean J Clin Psychol. 1997;16(1):185-197.

34. Lim S-U, Lee E-H, Hwang S-T, Hong S-H, Kim J-H. The beck depression inventory-: psychometric properties in Korean adult populations. Korean J Clin Psychol. 2019;38(3):300-307. doi:10.15842/kjcp.2019.38.3.003

35. Lee H-K, Lee E-H, Hwang S-T, Hong S-H, Kim J-H. Psychometric properties of the Beck Anxiety Inventory in the community-dwelling sample of Korean adults. Korean $J$ Clin Psychol. 2016;35 (4):822-830. doi:10.15842/kjcp.2016.35.4.010

36. Blake DD, Weathers FW, Nagy LM, et al. The development of a clinician-administered PTSD scale. J Trauma Stress. 1995;8 (1):75-90. doi:10.1002/jts.2490080106

37. Eun HJ, Kwon TW, Lee SM, Kim TH, Choi MR, Cho SJ. A study on reliability and validity of the Korean version of impact of event scale-revised. J Korean Neuropsychiatr Assoc. 2005;44(3):303-310.

38. Weiss DS. The impact of event scale: revised. In: Cross-Cultural Assessment of Psychological Trauma and PTSD. Springer; 2007:219-238.

39. Buysse DJ, Reynolds CF, Monk TH, Berman SR, Kupfer DJ. The Pittsburgh sleep quality index: a new instrument for psychiatric practice and research. Psychiatry Res. 1989;28(2):193-213. doi:10.1016/0165-1781(89)90047-4

40. Kong N, Choi J, Seo WS. Evaluation of sleep problems or disorders using sleep questionnaires. Chronobiol Med. 2019;1(4):144-148. doi:10.33069/cim.2019.0028

41. Johns MW, New A. Method for measuring daytime sleepiness: the Epworth sleepiness scale. Sleep. 1991;14(6):540-545. doi:10.1093/ sleep/14.6.540

42. Cho YW, Lee JH, Son HK, Lee SH, Shin C, Johns MW. The reliability and validity of the Korean version of the Epworth sleepiness scale. Sleep Breath. 2011;15(3):377-384. doi:10.1007/s11325010-0343-6

43. Bastien $\mathrm{CH}$, Vallières $\mathrm{A}$, Morin $\mathrm{CM}$. Validation of the Insomnia Severity Index as an outcome measure for insomnia research. Sleep Med. 2001;2(4):297-307. doi:10.1016/S1389-9457(00)00065-4

44. Cho YW, Song ML, Morin CM. Validation of a Korean version of the insomnia severity index. J Clin Neurol. 2014;10(3):210. doi:10.3988/ jen.2014.10.3.210

45. Ha KS, Kwon JS, Lyoo IK, Kong SW, Lee DW, Youn T. Development and standardization process, and factor analysis of the computerized cognitive function test system for Korea adults. J Korean Neuropsychiatr Assoc. 2002;41(3):551-562.

46. Salum GA, Sato JR, Manfro AG, et al. Reaction time variability and attention-deficit/hyperactivity disorder: is increased reaction time variability specific to attention-deficit/hyperactivity disorder? Testing predictions from the default-mode interference hypothesis. ADHD Atten Deficit Hyperact Disord. 2019;11(1):47-58. doi:10.1007/s12402-018-0257-x 
47. Levy F, Pipingas A, Harris EV, Farrow M, Silberstein RB. Continuous performance task in ADHD: is reaction time variability a key measure? Neuropsychiatr Dis Treat. 2018;14:781. doi:10.2147/ NDT.S158308

48. Epstein JN, Hwang ME, Antonini T, Langberg JM, Altaye M, Arnold LE. Examining predictors of reaction times in children with ADHD and normal controls. J Int Neuropsychol Soc JINS. 2010;16 (1):138. doi:10.1017/S1355617709991111

49. Collop NA, Anderson W, Boehlecke B, et al. Clinical guidelines for the use of unattended portable monitors in the diagnosis of obstructive sleep apnea in adult patients. J Clin Sleep Med. 2007;3 (7):737-747.

50. Berry RB, Gamaldo CE, Harding SM, et al. AASM scoring manual version 2.2 updates: new chapters for scoring infant sleep staging and home sleep apnea testing. J Clin Sleep Med. 2015;11(11):1253-1254. doi: $10.5664 /$ jesm. 5176

51. Lies J, Jobson L, Mascaro L, Whyman T, Drummond SPA. Postmigration stress and sleep disturbances mediate the relationship between trauma exposure and posttraumatic stress symptoms among Syrian and Iraqi refugees. J Clin Sleep Med. 2021;17(3):479-489. doi: $10.5664 /$ jcsm. 8972

52. Mysliwiec V, Brock MS, Creamer JL, O'Reilly BM, Germain A, Roth BJ. Trauma associated sleep disorder: a parasomnia induced by trauma. Sleep Med Rev. 2018;37:94-104. doi:10.1016/j. smrv.2017.01.004

53. Brownlow JA, Miller KE, Gehrman PR. Insomnia and cognitive performance. Sleep Med Clin. 2020;15(1):71-76. doi:10.1016/j. jsmc.2019.10.002

54. Killgore WDS. Effects of sleep deprivation on cognition. In: Kerkhof GA, Dongen HPA, van BT, editors. Progress in Brain Research. Vol. 185. Elsevier; 2010:105-129. doi:10.1016/B9780-444-53702-7.00007-5

55. Krause AJ, Ben SE, Mander BA, et al. The sleep-deprived human brain. Nat Rev Neurosci. 2017;18(7):404-418. doi:10.1038/ nrn.2017.55

56. Olaithe M, Bucks RS, Hillman DR, Eastwood PR. Cognitive deficits in obstructive sleep apnea: insights from a meta-review and comparison with deficits observed in COPD, insomnia, and sleep deprivation. Sleep Med Rev. 2018;38:39-49. doi:10.1016/j.smrv.2017.03.005

57. Park Y-M. Chronotype is associated with emotional dysregulation influenced by childhood trauma: a retrospective study. Chronobiol Med. 2019;1(1):21-25. doi:10.33069/cim.2018.0008

58. Lewis C, Lewis K, Kitchiner N, Isaac S, Jones I, Bisson JI. Sleep disturbance in post-traumatic stress disorder (PTSD): a systematic review and meta-analysis of actigraphy studies. Eur J Psychotraumatol. 2020;11(1):1767349. doi:10.1080/20008198.20 20.1767349
59. Zhang Y, Ren R, Sanford LD, et al. Sleep in posttraumatic stress disorder: a systematic review and meta-analysis of polysomnographic findings. Sleep Med Rev. 2019;48:101210. doi:10.1016/j. smrv.2019.08.004

60. Aserinsky E, Kleitman N. Regularly occurring periods of eye motility, and concomitant phenomena, during sleep. Science (80-). 1953;118(3062):273LP-274. doi:10.1126/science.118.3062.273

61. Germain A. Sleep disturbances as the hallmark of PTSD: where are we now? Am J Psychiatry. 2013;170(4):372-382. doi:10.1176/appi. ajp.2012.12040432

62. Seo J, Oliver KI, Daffre C, Moore KN, Lasko NB, Pace-Schott EF. In trauma-exposed individuals, self-reported hyperarousal and sleep architecture predict resting-state functional connectivity in frontocortical and paralimbic regions. Biol Psychiatry Cogn Neurosci Neuroimaging. 2019;4(12):1059-1069. doi:10.1016/j.bpsc.201 9.06.013

63. Vanderheyden WM, Poe GR, Liberzon I. Trauma exposure and sleep: using a rodent model to understand sleep function in PTSD. Exp Brain Res. 2014;232(5):1575-1584. doi:10.1007/s00221-014-3890-4

64. Ackermann S, Rasch B. Differential effects of non-REM and REM sleep on memory consolidation? Curr Neurol Neurosci Rep. 2014;14 (2):430. doi:10.1007/s11910-013-0430-8

65. Vanderheyden WM, George SA, Urpa L, Kehoe M, Liberzon I, Poe GR. Sleep alterations following exposure to stress predict fear-associated memory impairments in a rodent model of PTSD. Exp Brain Res. 2015;233(8):2335-2346. doi:10.1007/s00221-0154302-0

66. Nkomo TS. Exploring Congolese refugees and asylum seekers'survival strategies in South Africa: implication for social work practice. J Hum Behav Soc Environ. 2019;29(4):499-518. doi:10.1080/ 10911359.2018.1556142

67. DeGutis J, Esterman M, McCulloch B, Rosenblatt A, Milberg W, McGlinchey R. Posttraumatic psychological symptoms are associated with reduced inhibitory control, not general executive dysfunction. $J$ Int Neuropsychol Soc. 2015;21:342-352. doi:10.1017/ S1355617715000235

68. Fortenbaugh FC, Corbo V, Poole V, et al. Interpersonal early-life trauma alters amygdala connectivity and sustained attention performance. Brain Behav. 2017;7(5):e00684. doi:10.1002/brb3.684

69. Miller KE, Brownlow JA, Gehrman PR. Sleep in PTSD: treatment approaches and outcomes. Curr Opin Psychol. 2020;34:12-17. doi:10.1016/j.copsyc.2019.08.017

70. Byun J-H, Kim KT, Moon H, Motamedi GK, Cho YW. The first night effect during polysomnography, and patients' estimates of sleep quality. Psychiatry Res. 2019;274:27-29. doi:10.1016/j.psychres.20 19.02.011

\section{Publish your work in this journal}

Nature and Science of Sleep is an international, peer-reviewed, open access journal covering all aspects of sleep science and sleep medicine, including the neurophysiology and functions of sleep, the genetics of sleep, sleep and society, biological rhythms, dreaming, sleep disorders and therapy, and strategies to optimize healthy sleep.
The manuscript management system is completely online and includes a very quick and fair peer-review system, which is all easy to use. Visit http://www.dovepress.com/testimonials.php to read real quotes from published authors. 